East African Medical Journal Vol. 80 No. 10 October 2003

DISSEMINATED CRYPTOSPORIDIOSIS IN TURKEY: CASE REPORT

D. Dirim, MD, H. Dagci, MD, and N. Turgay, MD, Associate Professor, Ege University, Medical Faculty, Department of Parasitology, Bornova, Izmir, 35100, Turkey

Request for reprints to: Dr. N. Turgay, Ege University, Medical Faculty, Department of Parasitology, Bornova, Izmir, 35100, Turkey

\title{
DISSEMINATED CRYPTOSPORIDIOSIS IN TURKEY: CASE REPORT
}

\author{
D. DIRIM, H. DAGCI and N. TURGAY
}

\begin{abstract}
SUMMARY
Cryptosporidium $s p$ is a protozoan that displays an intracellular settlement primarily in the intestinal systems of humans and can result in diarrhoea. Undernourished children and persons with immunosuppression in developing countries are especially vulnerable to infection with this parasite. A 12-month-old female presented at Ege University, Faculty of Medicine, Department of Paediatrics with complaints of fever, diarrhoea, respiratory distress and growth- development retardation was diagnosed with CD40 deficiency (Hyper IgM Type 3). During the one year investigation process of the case with chronic diarrhoea and necrotic pneumonia, Cryptosporidium sp oocysts were found in nine of the 22 faecal examinations and also in transtracheal aspiration liquid examined using the Kinyoun Acid-fast staining method. In conclusion, it is thought that cryptosporidiosis should also be considered in the distinct diagnosis of immunodeficient infants who are presented with respiratory and gastrointestinal system complaints.
\end{abstract}

\section{INTRODUCTION}

Human infection with Cryptosporidium parvum has been reported from over 90 countries in six continents and the prevalence of the infection found to be higher in developing countries. Children, the undernourished and persons with immunodeficiency are more vulnerable to $C$. parvum infection (1).

Clinical manifestations of Cryptosporidium $s p$. infection change depending on the immune system of the host. Symptomatic cryptosporidiosis in a immunocompetent host is characterised with diarrhoea lasting about two weeks, abdominal cramps, nausea, fever and weight loss (2). However, in immunosuppressed patients, it may cause diarrhoea resembling to cholera up to 20 litters daily, leading to a life threatening state and the parasite may settle in the extraintestinal locations including the lungs, bile and pancreatic ducts, stomach and middle ear (4).

The diagnosis of cryptosporidiosis is established through the detection of oocysts of 4-6 $\mu$ m diameter in stool, bronchoalveolar lavage liquid, transtracheal aspiration liquid and bile specimens using various staining methods such as the Kinyoun Acid-fast and Modified ZiehlNeelsen(1).

The objective of this study was to search for Cryptosporidium sp in a 12-month- old female case who was diagnosed with CD40 deficiency (Hyper IgM Type 3) (5) following an examination of stool and respiratory system specimens.

\section{CASE REPORT}

A 12-month- old female patient with respiratory distress and growth-development retardation was admitted to Ege University, Faculty of Medicine, Department of Paediatrics with a complaint of high fever. While the baby girl exhibited a normal growth and development during the first nine months, she was then hospitalised due to respiratory syncytial virus (RSV) pneumonia and given mechanical ventilations. Physical examination of the patient, who had tachypnoea and intercostal contractions revealed that the liver was $5 \mathrm{~cm}$ below the costal region. It was further observed that the patient had chronic watery diarrhoea during hospitalisation.

Leukocytosis $\left(20.2-437 \times 10^{9} / \mathrm{L}\right)$, eosinophilia (>5×109/ $\mathrm{L})$, medium-level anaemia (10.3 gr/dl) and thrombocytosis $\left(828 \times 10^{9} / \mathrm{L}\right)$ were detected in the patient and alkaline phosphates (1858 U/L) and y glutamyl transpeptidase (558 $\mathrm{U} / \mathrm{L}$ ) values were found to be high. Abdominal ultrasonography showed a dilatation in the intrahepatic bile ducts as well as periportal hyperechogenity. While the biopsy of the liver revealed a cystic dilatation in the bile ducts, and eosinophilia and polymorphonuclear Iymphocyte (PML) infiltration in periductal regions, pulmonary fibrosis, atelectasis and bronchiectasis were found in computerised tomography (CT) of the thorax. Excessive eosinophilia was detected in TTA. At the time of hospitalisation, the infant was determined to have severe necrotic pneumonia. Tracheostomy was administered. Following the severe manifestation of a laryngo-tracheobronchitis. Pseudomonas aeruginosa was found in blood and pleural effusion cultures.

Following immunological and genetic examinations, the patient was diagnosed with autosomal recessive Hyperimmunoglobulin M (HIGM3) associated with CD40 deficiency (5). The IgM value of the patient, whose serum IgG and IgA values were substantially reduced, was found to be within normal limits $(80 \mathrm{mg} / \mathrm{dl})$. 
Figure 1

Faecal smear stained with Kinyoun Acid-fast stain shows oocyst of Cryptosporidium sp. (original magnification $x 1000)$

\section{Figure 2}

Transtracheal aspiration liquid smear shows oocyst of Cryptosporidium sp. stained with Kinyoun Acid-fast stain, surrounded by respiratory system epithelial cells (original magnification x1000)

Parasitological Examinations: TTA liquid of the patients was rotated in a cytocentrifuge for 10 minutes at $1500 \mathrm{rpm}$; then the preparates were stained and examined using Giemsa, Gram -Weigert and Kinyoun Acid -fast staining methods(7). The stool specimens were smeared after employing wet- mount, Lugol 's and Ritchie methods, stained using Trichrome and Kinyoun Acid-fast staining methods and examined for a total of 22 times at different intervals during a period of one year. Moreover, stool specimens taken from the whole family were examined thoroughly using the same methods.

Pneumocystis carinii was not encountered in the TTA sample examined by means of Giemsa and Gram -Weigert staining methods, whereas examination by
Kinyoun Acid -fast staining method revealed the presence of Cryptosporidium $s p$.

Cryptosporidium $s p$ was detected in nine of the 22 stool specimens examined by Trichrome and Kinyoun Acid -fast staining methods and Endolimax nana was found in one of these nine stools. Stool examinations of the family, however, revealed no sign of parasites.

\section{DISCUSSION}

While cryptosporidiosis basically causes asymptomatic or limited diarrhoea in immunocompetent persons, it is a parasitic infection that leads to diarrhoea that follows a cholera-like course with dehydration and may even result in death in immunodeficient persons(6).

According to data obtained from various studies carried out in the world, the prevalence of cryptosporidiosis has been reported as 1-2\% in Europe, $0.6-4.3 \%$ in North America, and 3-20\% in Asia, Australia, Africa, Central and South America(6). Cryptosporidiosis has a significant place in childhood diarrhoeas and makes a peak between the ages 1-5 years. The prevalence among children with diarrhoea is $1-3 \%$ in industrialised countries, whereas it ranges between $4-7 \%$ in developing countries(4). There is no clear information related to the general prevalence in both adults and children Cryptosporidium infection; most of the data available are in the form of results of published studies.

Cryptosporidiosis is one of the opportunistic infections encountered in immunosuppressed persons. This parasite basically flourishes with the suppression in cellular immunity and is particularly found in congenital immune deficiencies displaying cellular immunity defects(8). In HIGM3, a defect is observed in $\mathrm{T}$ cell activation associated with CD40 deficiency in macrophage and dendritic cells and CD40 ligand (CD40L) interaction. Therefore, these opportunistic infections are not encountered in type 2 hyper IgM syndrome associated with Activation Induced Diaminase (AID) mutation although immunoglobulins are low. Chronic and disseminated infections occurring during opportunistic infections caused by Cryptosporidium sp. are often found in Type 1 with CD40L deficiency and Type 3 with CD40 deficiency(9-11). Chronic cryptosporidiosis may also be seen in the course of AIDS, which progresses with CD4 deficiency(12). Furthermore, chronic cryptosporidiosis is known to be a cause of growth development retardation in children(6), and it is also believed that this infection is one of the factors responsible for the growth - development retardation in our case.

While Cryptosporidium $s p$ settles primarily in the gastrointestinal systems of persons having normal immune systems, causing diarrhoea, it can lead to various clinical manifestations (gastritis, sinusitis, cholecystitis, pancreatitis, hepatitis) in extraintestinal sites like the respiratory system in immunodeficient 
patients $(6,13,15)$. In a study conducted by Clavel et al.(16) 57 respiratory cryptosporidiosis cases were reported since 1980 and that all of these cases were immunodeficient patients(16-19). Cases that progress with respiratory distress cause chronic bronchial symptoms and diagnosis is established through detection of Cryptosporidium $s p$ in various respiratory specimens from broncho-alveolar lavage, sputum and bronchial biopsy(20). Following the CT evidence of the case such as pulmonary fibrosis, atelectasis and bronchiectasis, abundant eosinophilia and Cryptosporidium sp oocysts were determined during TTA material examination, leading us to a conclusion that this severe laryngotracheobronchial manifestation was associated with cryptosporidiosis. Another extraintestinal localization of cryptosporidiosis in immunodeficient patients is the biliary tract. Forbes et al. found that Cryptosporidium $s p$ in 13 out of 20 cases, and estimated that up to one in six of all the cases of AIDS-related cryptosporidiosis may also have sclerosing cholangitis(21). A patient with AIDS reported by Teixidor et al. was found to have sclerosing cholangitis that was progressing with cryptosporidiosis-related cholecystitis. It was further stated that this was often seen in patients with chronic diarrhoea, with elevated serum alkaline phosphates and glutamyl transpeptidase enzymes which might indicate of hepatic damage. In our case, the ultrasonography indicated the presence of irregularly dilated intrahepatic bile ducts. The fact that elevated alkaline phosphates and glutamyl transpeptidase enzymes, with abdominal ultrasonographic evidence of periportal hyperechogenity and dilatation in the intrahepatic bile ducts and cystic dilation in the bile ducts, eosinophilia and polymorphonuclear Iymphocyte (PML) infiltration in periductal areas in the liver biopsy material lead us to think that there could be a biliary system invasion associated with cryptosporidiosis in our case.

Having considered this case that carries a great importance as it is the first recorded case of disseminated cryptosporidiosis in Turkey, it has been concluded that cryptosporidiosis should also be searched for while establishing the final diagnosis of infections that progress with chronic diarrhoea and respiratory distress in children with immune deficiency and growthdevelopment retardation.

\section{REFERENCES}

1. Fayer, R., Morgan U. and Upton S.J. Epidemiology of Cryptosporidium transmission, detection and identification. Int. J. Paras. 2000; 30:1305-1322.

2. Markell E.K., John D.T. and Krotoski W.A. Lumen Dwelling Protozoa in "Markell and Voge's Medical Parasitology', Philadelphia:Saunders Company. 1999. P24-89.
3. Clark, D.P. New insights into human cryptosporidiosis. Clin. Micro. Rev. 1999; 554-568.

4. Farthing, M.J.G., Cevallos A.M., Kelly P. Intestinal Protozoa in Manson's Tropical Diseases. London: W.B. Saunderss Company. 1996. P1255-1298.

5. Kutukculer, N, Moratto, D., Aydinok, Y., et al. Disseminated cryptosporidium infection in an infant with hyper-IgM syndrome caused by CD40 deficiency. J. Pediatr. 2003; 42:194-196.

6. Garcia, L.S. and Bruckner D.A. Macroscopic and Microscopic Examination of Fecal Specimens in "Diagnostic Medical Parasitology" Washington DC: American Society for Microbiology. Press. P 49-74. 1993.

7. Lawrence, R. and Orihel T.C. Procedures for AIDS-Related parasitic infections. Parasites. Am. Soc. Clin. Patho. 1987; 91.

8. Stiehm, E.R., Chin, T.W., Haas, A. and Peerless, A.G. Infectious complications of the primary immunodeficiencies. Clin Immunol. 1986; 40:69-86.

9. Ramesh, N., Geha R.S., and Notarangelo, L.D. CD40 ligand and the hyper-IgM, syndrome in "Primary Immunodeficiency Diseases" New York: Oxford University. Press. 1999.P. 233249.

10. Jo, E.K., Kim H.S., Lee, M.Y., et al. X-linked hyper-IgM syndrome associated with Cryptosporidium parvum and Cryptococcus neoformans infections: the first case with molecular diagnosis in Korea. J. Korean. Med. Sci. 2002; 17:116-120.

11. Levy, J. Espanol-Boren, T., Thomas, C., et al. Clinical spectrum of X-linked hyper-IgM syndrome. J. Pediatr. 1997; 131:47-54

12. Lebbad, M., Norrgren, H., Naucler, A., et al. Intestinal parasites in HIV-2 associated AIDS cases with chronic diarrhoea in Guinea Bissau. Acta. Trop. 2001; 80:45-49.

13. Clemente, C.M., Caramori, C.A., Padula, P., and Rodrigues, M.A. Gastric cryptosporidiosis as a clue for diagnosis of the acquired immunodeficiency syndrome. Arq. Gastroentero. 2000; 37:180-182.

14. Davis, J.J. and Heyman, M.B. Cryptosporidiosis and Sinusitis in an Immunodeficient Adolescent. J. Infec. Dis. 1988; 158:649.

15. Teixidor H.S, Godwin T.A. and Ramirez E.A. Cryptosporidiosis of Biliary Tract in AIDS. Radiology. 1991; 180:51-56.

16. Clavel, A., Arnal, A.C., Sanchez, E.C. et al. Respiratory cryptosporidiosis: case series and review of the literature. Infection. 1996; 24:341-346.

17. Moore, J.A., and Frenkel, J.K. Respiratory and Enteric Cryptosporidiosis in Humans. Arch. Pathol. Lab. Med. 1991; 115:1160-1162.

18. Travis W.D., Schmidt K., MacLowry, J.M., et al. Respiratory Cryptosporidiosis in a patient with malignant lymphoma. Arch. Pathol. Lab.Med. 1990; 114:519-523.

19. Hojlyng, N. and Jensen, B.N. Respiratory Cryptosporidiosis in HIV-Positive Patients. Lancet, 1988; 12:590-591.

20. Stern, J.B., Antoine M., Roux P., Mayaud C. and Cadranel J. Pulmonary in a patient with AIDS. Rev. Mal. Resp. 1998; 15:549-553.

21. Forbes, A, Blanshard, C., and Gazzard, B. Natural history of AIDS related sclerosing cholangitis: a study of 20 cases. Gut. 1993; 34:116-121. 